\title{
Philosophiques
}

\section{Critique d'une critique (Réponse à Louise Marcil-Lacoste)}

\section{Claude Collin}

Volume 8, numéro 1, avril 1981

URI : https://id.erudit.org/iderudit/203155ar

DOI : https://doi.org/10.7202/203155ar

Aller au sommaire du numéro

Éditeur(s)

Société de philosophie du Québec

ISSN

0316-2923 (imprimé)

1492-1391 (numérique)

Découvrir la revue

Citer ce document

Collin, C. (1981). Critique d'une critique (Réponse à Louise Marcil-Lacoste).

Philosophiques, 8(1), 149-166. https://doi.org/10.7202/203155ar d'utilisation que vous pouvez consulter en ligne.

https://apropos.erudit.org/fr/usagers/politique-dutilisation/ 


\title{
INTERVENTIONS
}

\section{CRITIQUE D'UNE CRITIQUE}

\author{
Réponse à Louise Marcil-Lacoste \\ par Claude Collin
}

Le compte rendu critique de Louise Marcil-Lacoste sur l'Expérience philosopbique ${ }^{1}$, paru dans un récent numéro de la revue Dialogue $\left(\mathrm{n}^{\circ} 2,1980\right)$, soulève des questions importantes concernant l'élaboration d'une didactique objective de la philosophie. La principale faiblesse de ce compte rendu s'enracine dans une certaine ambiguïté concernant les notions fondamentales de cette science encore toute jeune, qui n'en est d'ailleurs qu'au stade de la construction de modèles théoriques.

Bien que ces notions fondamentales de la didactique aient été précisées dans l'Enseignement de la philosopbie ${ }^{2}$ et chez des auteurs comme Buyse, Mialaret, Bloom, Bruner, etc. ${ }^{3}$, il me semble nécessaire d'y revenir, ne serait-ce que pour resituer dans leur véritable perspective les problèmes actuels de l'enseignement de la philosophie. Il s'agit plus particulièrement des notions de faits pédagogiques, d'hypothèse méthodologique, de comportement et de modèles théoriques.

Pour plus de clarté, j'aborderai ces questions en suivant de près le texte de Lacoste, qui se divise en deux parties: la première tente de résumer l'essentiel de l'Expérience philosophique, tout en soulevant quelques difficultés; la seconde est une critique de la méthode de recherche utilisée dans cet essai de didactique expérimentale.

1. Claude Collin, l'Expérience philosophique, essai de didactique expérimentale, Bellarmin, 1978.

2. Claude Collin et Z. Ozana, l'Enseignement de la philosophie, Fides, 1974.

3. Les références bibliographiques des ouvrages de ces auteurs sont indiquées ci-dessous, p. 00 et 00 . 


\section{PREMIÈRE PARTIE}

\section{Le contenu de l'Expérience philosophique}

Pour rendre compte du contenu de l'Expérience philosopbique, Lacoste le compare tout d'abord au premier ouvrage de l'auteur, réalisé en collaboration avec $Z$. Osana; ensuite, elle soulève quelques difficultés sur une partie de l'hypothèse de départ (la spécificité de la philosophie); enfin, elle exprime ce qui lui semble la thèse centrale de l'ouvrage.

1) De l'Enseignement de la philosophie à l'Expérience philosopbique

Le compte rendu cherche d'abord à préciser l'évolution d'un essai à l'autre:

a) L'Enseignement de la philosophie, lit-on, s'intéressait aux facteurs contrôlables de l'enseignement de la philosophie. L'Expérience philosophique met l'accent sur l'étudiant, sur les caractéristiques psychologiques du cégépien et soutient avoir établi un modèle de fonctionnement mental basé sur de telles caractéristiques.

\section{REMARQUES}

Cette différence entre les deux essais n'est pas exacte. Dans les deux essais nous affirmons la nécessité d'une connaissance de l'étudiant réel, dans la perspective d'une recherche scientifique, en didactique de la philosophie. Qu'il suffise de citer les passages suivants du premier essai:

Plus particulièrement, la didactique expérimentale devra établir elle-même, comme l'affirme Buyse, les données psychologiques, par exemple, les constantes des opérations mentales des étudiants, ce qu'ils savent faire, ce qu'ils peuvent faire et ce qu'ils font d'un point de vue psychologique ${ }^{4}$. (L'Enseignement de la philosophie, p. 20.)

... quelle que soit la valeur del'idéologie, le problème pédagogique reste entier, aussi longtemps que l'on ne parvient pas à démontrer, à l'aide de faits, comment les étudiants fonctionnent mentalement, face à l'apprentissage de la philosophie. (Ibid., p. 4.) 
Le but de cette épreuve était donc d'étudier le comportement des étudiants et leur réaction face à un problème philosophique. (Ibid., p. 14.)

Il ne s'agit donc pas d'une question d'accent, comme l'affirme Lacoste, mais d'une exigence didactique que l'on retrouve dans les deux essais. La différence entre eux, sur ce point précis, est indiquée à la page 14 de l'Expérience philosophique:

En publiant notre premier essai, nous ne disposions pas de faits pédagogiques significatifs suffisants pour cerner ce problème avec précision et d'une façon complète. Nous avons l'impression d'avoir progressé en ce sens, en poursuivant notre recherche ( p. 14-15).

Il convient de noter qu'il ne s'agit pas, comme le prétend Lacoste, de mettre l'accent sur l'étudiant, sur les caractéristiques psychologiques du cégépien; il s'agit de connaître le comportement mental de l'étudiant EN ÉTAT D'APPRENTISSAGE de la philosophie, c'est-à-dire le comportement en relation avec une tâche philosophique. Il ne relève pas de la didactique de considérer un comportement en soi, mais en tant qu'il peut constituer un fait pédagogique. (Voir la notion de fait pédagogique, dans l'Enseignement de la philosophie, p. 10-13.)

b) L'évolution majeure d'un essai à l'autre, écrit Lacoste, réside en ceci que Collin affirme, avec plus de fermeté et d'insistance, que la formulation, par l'étudiant, de l'expérience vécue, doit être le point de départ de la réflexion philosophique.

\section{REMARQUES}

Le choix de l'expérience vécue, comme point de départ à la réflexion philosophique, n'est qu'un choix parmi d'autres possibles et que nous avons fait dans les deux essais.

Les sources d'information possibles pour le philosophe, écrivionsnous dans l'Enseignement de la philosophie, semblent illimitées. Nous avons privilégié celle qui nous paraissait la plus apte à susciter un intérêt soutenu de la part de tout étudiant appartenant au secteur professionnel ou au secteur général, et la mieux appropriée pour lui permettre d'évoluer selon ses capacités réelles dans l'approfondissement d'une philosophie personnelle (p. 35).

Remarquons que, dans le second essai, ce choix figure dans l'introduction, parmi les six notions et raisons d'être de l'hypo- 
thèse de travail formulée au début de la recherche (voir p. 19-35, de l'Expérience philosopbique). Il est donc inexact d'affirmer que c'est là une évolution majeure. À mon avis, l'évolution d'un essai à l'autre serait plus adéquatement décrite en termes de suite, d'approfondissement et de développement.

Il n'existe aucune différence dans l'esprit et la méthode utilisée. Nous aurions pu inclure tous les tableaux indiquant ce qu'ont fait les étudiants lors des différentes épreuves qui nous ont conduit à la description du modèle du fonctionnement mental de l'étudiant en rapport avec les différentes tâches (comme nous l'avions fait quant à l'analyse conceptuelle, dans le premier ouvrage). Nous avons préféré décrire une théorie basée sur les faits pédagogiques les plus significatifs. Nous avons procédé ainsi afin d'alléger la présentation du travail. Mais l'esprit et la méthode sont dans une continuité évidente d'un essai à l'autre.

En termes d'approfondissement et de développement, le second essai constitue, aux yeux du lecteur, un progrès notable. Le seul fait de pouvoir identifier le problème fondamental de l'enseignement de la philosophie marque un progrès appréciable, puisqu'il suppose un long cheminement et de nombreuses études préalables. Dans le premier essai, nous écrivions en conclusion: "L'ébauche expérimentale nous montre qu'on ne peut songer à étudier les problèmes pédagogiques que si l'on se penche soigneusement sur l'apprentissage systématique et significatif des thématiques, du vocabulaire et des concepts philosophiques" (p. 75). Or dans le second essai, nous pouvons formuler le problème fondamental de la façon suivante: "Comment l'étudiant peut-il parvenir à vivre des expériences philosophiques de type achevé et ainsi développer un esprit philosophique?" (L'Expérience philosopbique, p. 15).

Pour être en mesure de poser en ces termes le problème, cela suppose déjà la production de modèles théoriques qui ne soient pas arbitraires, c'est-à-dire construits in abstracto, mais qui impliquent de nombreuses études de faits pédagogiques dans l'esprit de la méthode de recherche proposée dans le premier essai. Ce dernier, d'ailleurs, ne comportait pas d'élaboration théorique, tandis que nous y consacrons 80 pages (p. 39-119) dans l'Expérience philosophique. 
De plus, le premier essai ne comportait pas de discussion de problèmes didactiques pratiques (comme, par exemple, celui de la détermination du concept central d'une expérience), alors que, dans le second essai, nous abordons plusieurs de ces problèmes, de la page 123 à la page 150 . Ajoutons que le premier essai présentait une étude sous forme d'expérimentation de la méthode didactique proposée alors; or les résultats de cette étude nous ont conduit à préciser davantage les composantes de la méthode didactique. Ceci fut à l'origine de l'analyse de l'expérience, de l'analyse propositionnelle et de la problématisation, que nous retrouvons dans le second ouvrage. Il y a là une évolution considérable, qu'il serait trop long d'expliquer en détails, mais qui nous a permis d'apporter un élément de mesure de la capacité d'analyse. Il s'agit d'une première quantification en didactique philosophique. Cela méritait sûrement d'être signalé.

Il serait fastidieux de reprendre ici un à un tous les approfondissements et leurs aboutissements. Mais on ne peut certes les résumer par les termes "plus de fermeté et plus d'insistance à proposer la formulation de l'expérience vécue comme point de départ à la réflexion philosophique», comme l'affirme Lacoste. Cet aspect n'est qu'une des composantes de l'hypothèse de départ de la recherche.

Il ne faut pas confondre la conclusion du chapitre théorique sur l'expérience vécue des étudiants avec cette composante. Cette conclusion ne porte pas sur LA FORMULATION de l'expérience vécue, mais sur la nécessité d'avoir recours à l'expérience à tous les moments de la réflexion philosophique.

Cette conclusion se lit ainsi: "L'expérience est un point de départ approprié à la réflexion philosophique, puisque celle-ci se présente comme une forme achevée d'expérience. La réflexion philosophique est une expérience qui dépend des expériences antérieures» (p. 71). Puis plus loin: «Il faut toujours référer au vécu, à ce que l'étudiant sait pour l'avoir vécu» (p. 73). Et ensuite: «L'expérience est un apprentissage; on apprend par l'expérience et l'on comprend par les expériences réfléchies» (p. 72). Enfin: «Toute réflexion est expérience vécue et tend naturellement vers un type achevé» (p. 72). 
Ces affirmations doivent être comprises dans le cadre de l'élaboration d'un modèle théorique de la réflexion, appuyé sur des faits. Ce que nous avons fait, ici, fut d'appliquer didactiquement au domaine de la philosophie ce qui est connu des processus d'apprentissage intellectuel. Si l'on comprend bien cela, on voit mieux pourquoi nous croyons que notre méthode didactique (y compris la formulation de l'expérience vécue comme point de départ) n'est pas, à nos yeux, la seule valable. Car «toute réflexion est expérience vécue», et l'apprentissage des concepts qui se réalise de toutes sortes de façons est déjà une expérience, qu'elle soit obtenue par voie d'exposition ou de découverte. Mais il ne faudrait pas confondre cela avec LA FORMULATION d'une expérience vécue.

\section{2) Les difficultés soulevées}

\section{Remarque préliminaire}

Avant d'aborder ce second point, je relève un bref passage, qui a sans doute échappé à l'auteur de ce compte rendu:

Collin critique toute didactique dont le point de départ serait pris du côté de la culture achevée.

Nous consacrons six pages (21-27) à la description des modes de détermination des points de départ à l'enseignement de la philosophie, en expliquant en quoi celui que nous choisissons differe des points de départ «traditionnels». À moins que l'on interprète ce terme dans un sens péjoratifs, il n'y a pas de raison de penser que nous essayons de les discréditer.

Il serait sans doute possible d'évaluer expérimentalement ces différents points de départ que l'on pourrait appeler traditionnels et les méthodes didactiques qui y sont rattachées (p. 22).

Nous sommes donc bien loin de porter un jugement de valeur sur ces points de départ. D'ailleurs, la méthode didactique que nous proposons se présente comme une méthode de travail intellectuel, un outil de recherche que l'on propose aux étudiants. «Elle doit être complétée par des cours magistraux bien structurés», disions-nous dans l'Enseignement de la philosopbie (p. 18).

Le seul doute soulevé a trait à un élément de la méthode sartrienne, et non à son point de départ: «Utilisée telle quelle, 
cette méthode (de Sartre) néglige PEUT-ÊTRE un aspect important de la réflexion philosophique, c'est-à-dire la critique de la pensée» (p. 26).

L'une des conclusions générales de l'Expérience pbilosophique est formulée comme suit: «La méthode didactique à laquelle nous sommes parvenu n'a pas la prétention d'être la seule valable. Bien au contraire; l'étudiant a sans doute besoin, en raison de la variété et de la pluralité des courants de pensée de notre société, de recevoir un enseignement procédant selon diverses méthodes» ( $p$. 193). Il est donc évident que l'affirmation de Lacoste est tout à fait dépourvue de fondement.

\section{Remarques sur la spécificité de la philosophie}

Abordons maintenant l'hypothèse de la spécificité de la réflexion philosophique qui serait, selon Lacoste, insuffisamment justifiée.

Cette question de la spécificité de la philosophie est traitée dans l'introduction de l'Expérience philosopbique comme l'une des composantes de l'hypothèse de travail que l'on trouve à la page 18. Déjà, dans l'Enseignement de la philosopbie, nous expliquions pourquoi, dans l'élaboration d'une didactique objective, il était nécessaire de proposer une hypothèse de ce genre au départ:

L'activité pédagogique de l'enseignant exerçant sa profession d'enseignant de la philosophie, présuppose qu'il connait sa matière de telle sorte qu'il puisse transmettre à la fois le contenu et l'esprit de sa science (savoir pour lui-même résoudre les problèmes pertinents à sa science). Il doit savoir ce qu'est la philosophie d'un point de vue psychologique, c'est-à-dire l'aspect psychologique de l'opération concernée. À quelle sorte d'activité va-t-il soumettre ses étudiants? Il lui faut présupposer une manière mentale de procéder, propre à la discipline qu'il doit enseigner (p. 21).

On doit savoir à quelle sorte d'apprentissage seront soumis les étudiants qui entreprendront l'étude des éléments de la philosophie (p. 7).

Ce n'est qu'à ce moment, et en fonction de cet objectif, que leurs réactions prendront un sens déterminant sur le plan pédagogique (p. 7).

Il faut comprendre le sens et la portée d'une telle hypothèse. Dans une optique didactique, la spécificité de la philosophie n'est 
pas une question de méthode philosophique: elle réfere plutôt à des pérations mentales spécifiques, que l'on retrouve dans pratiquement toutes les philosophies selon un ordre et un agencement différent. C'est pourquoi nous pouvons noter, dans l'Expérience philosopbique:

Le problème didactique consiste à savoir comment favoriser, chez l'étudiant réel, la maîtrise des opérations mentales qu'effectue le philosophe, lorsqu'il réfléchit à la manière d'un philosophe (p. 34).

Puis nous ajoutons, pour préciser encore le sens de cette hypothèse:

On peut différer d'opinion sur le sens et la valeur de l'expérience vécue, sur les procédés d'analyse et sur le principe de vérification. Mais on ne peut nier l'importance de ces procédés dans l'élaboration de la pensée philosophique. Ces procédés sont indépendants de l'empirisme, de l'atomisme logique, du physicalisme, du marxisme professé à la manière de Jean-Paul Sartre. Ces procédés sont utilisés par tous les philosophes, selon la conception qu'ils s'en font (p. 34)

Ce qui signifie, en d'autres termes, que toute méthode d'enseignement implique une hypothèse consciente ou non sur la spécificité de la philosophie. Cela fait partie de l'art d'enseigner, et n'est-ce pas le rôle d'une didactique scientifique d'aider cet art à prendre mieux conscience de ses propres lois et ses propres techniques?

Or Lacoste affirme que cette hypothèse n'est pas suffisamment justifiée; je ne vois dans son argumentation aucun élément, aucune raison didactique. C'est par le biais de l'extension à tous les domaines de la culture de cette hypothèse, qu'elle soulève cette difficulté:

À ce sujet, il (Collin) fait une affirmation dont on aurait souhaité voir la justification: «Quel que soit le champ culturel spécifiant l'expérience vécue, il est toujours possible de préciser les distinctions qui séparent les expériences dites communes et celles qui réalisent pleinement le type de réflexion propre à un domaine spécifique.» Une telle thèse renvoie à la question de la spécificité de la philosophie, dans le contexte des divers domaines de la culture achevée. Mais à ce propos, Collin se refuse à une justification philosophique.

Il est bien certain que si la méthode de recherche utilisée implique une hypothèse sur la spécificité de la discipline à ensei- 
gner, (en l'occurrence un domaine qui ne manque pas d'ambiguïté en tant que discipline), cette hypothèse vaut, à fortiori, pour les autres domaines de la culture, et pour les mêmes raisons didactiques. Ce n'est que logique, dans l'élaboration théorique du modèle didactique, d'assumer cette assertion en cohérence avec l'hypothèse de départ.

Mais il y a plus. Cette extension aux autres domaines de la culture de cette question de la spécificité, dans le contexte cité par Lacoste, s'appuie sur les faits pédagogiques concernant l'expérience, en particulier les caractères «préconscient» et «transformant» de l'expérience vécue. Voici d'ailleurs ce que nous écrivions à ce sujet:

a) L'expérience est un procédé culturel dont on peut distinguer différentes étapes et différents degrés de réalisation. Le niveau commun se présente comme une jonction vivante de la réalité extérieure et de la personne transformant celle-ci, même à son insu, sur le plan des capacités et des comportements. (...)

b) Les expériences sont spécifiées en premier lieu par le vécu qu'elles constituent. ( . . . À ce point de vue, on peut affirmer que l'expérience est un mode de vie, (. . .) elle est le processus d'évolution de l'homme, puisqu'elle le change en ce qui le fait être ce qu'il est.

c) Ce qui spécifie le vécu est une totalité envisagée dans son aspect dynamique. (...)

d) Un dernier élément de spécification, difficilement isolable du concept totalisant, viendrait du procédé lui-même que ce concept exige pour devenir opératoire selon tel type d'évolution. En effet, pour être opératoire, il doit appartenir à tel champ de la culture, tant par sa signification que par la façon dont il a reçu ses notes déterminées (p. 98-99).

Afin d'expliciter ces affirmations, de la page 99 à la page 108, nous comparons les expériences telles qu'elles se présentent dans les différents domaines culturels (en ayant recours à l'occasion à saint Jean de la Croix, à Gaston Bachelard, à Georges Friedman), avec les différents niveaux que nous avons remarqués dans les expériences philosophiques des étudiants. Cette comparaison avec les expériences prises en d'autres domaines nous aide à mieux illustrer le processus lui-même de la philosophie. Mais cela est une autre question. 
Ce qu'il faut retenir, à notre sens, c'est que si des raisons didactiques justifient l'hypothèse de la spécificité de la philosophie, elles doivent valoir aussi, et pour les mêmes motifs, quant aux autres domaines de la culture.

\section{3) La thèse centrale de l'ouvrage}

La thèse centrale de l'Expérience philosophique, écrit Lacoste, est que l'expérience vécue de l'étudiant est et doit être le point de départ de la réflexion philosophique. À ce slogan des années récentes, Collin désire apporter des éléments factuels et empiriques.

\section{REMARQUES}

Soulignons que cette référence au «slogan des années récentes" vient en contradiction avec notre conception du travail scientifique en pédagogie. Nous avons toujours combattu les conceptions pédagogiques issues des tendances idéologiques, psychologiques et sociologiques, qui sont souvent à l'origine de modes passagères. Nous l'avons fait, en particulier, dans un article sur le rapport Roquet, publié en $1971^{4}$, et aussi dans l'Enseignement de la philosophie, p. 5 et 11. C'est la raison, d'ailleurs, pour laquelle nous avons mis beaucoup d'insistance à décrire la méthode de recherche scientifique et, plus particulièrement, le fait pédagogique qui est à la base de notre procédé de recherche.

Il faut dire que l'Expérience philosophique n'est pas et ne doit pas être considérée comme une thèse sur le point de départ de l'enseignement de la philosophie. Elle est une recherche qui tente, d'une part, d'identifier un certain nombre de problèmes propres à l'enseignement de la philosophie, et, d'autre part, d'appliquer au domaine de la philosophie ce qui est connu des processus de l'apprentissage intellectuel et de formuler les opérations à effectuer en termes d'objectifs de comportement.

Il existe, en effet, un certain nombre d'ouvrages scientifiques se rapportant au processus d'apprentissage intellectuel dont nous nous sommes inspiré et qui figurent dans les bibliographies des deux essais. L'application de ces connaissances à l'étude des

4. "Le rapport Roquet", dans l'Action Nationale, vol. LX, ${ }^{\circ} 8$, et "Le test perpe-philo", dans Prospectives, nov. 1971. 
problèmes pédagogiques de l'enseignement de la philosophie devrait constituer, dans notre contexte scolaire, un apport dont le temps déterminera l'importance et l'originalité. Mais si l'on tient à y découvrir une thèse centrale, il me semble, au moins, que celle-ci devrait porter sur les problèmes pédagogiques (non sur une hypothèse de départ à la recherche) et sur les modèles didactiques concernant la réalisation d'une expérience philosophique, puisque c'est là l'objectif même de la recherche, tel que cité plus haut.

Qu'il me soit permis de citer ici un auteur philosophe du Québec, jouissant d'une longue expérience de l'enseignement de la philosophie, qui faisait les affirmations suivantes dans son compte rendu sur l'Expérience philosophique, paru dans Le Devoir ${ }^{5}$ :

Le récent livre de Claude Collin, ( . . .) vient juste à point proposer une méthode d'initiation à la philosophie, fondée non point sur un enrégimentement des jeunes esprits, par l'imposition d'une idéologie militante; mais sur la nature même des processus philosophiques, en tout esprit qui réfléchit et qui élabore, selon cette discipline, les données de l'expérience humaine.

Une étude sur le terrain nous est donc présentée, une exploration (activité expérimentale) dans le domaine de la philosophie, ouverte au concret-qui-lui-arrive. Calqué sur le mouvement même de l'élaboration philosophique, le procédé didactique qui en découle se schématise comme suit:

La production de nombreux travaux d'élèves n'est pas la section la moins intéressante de ce court traité de pédagogie expérimentale en philosophie. Ils révèlent rigoureusement la démarche mentale indiquée. Dans ce livre, tout apparaît «d'expérience» (sensible ou intellectuelle): des premières données concrètes, à la problématisation et à la vérification.

\section{Et pour terminer, il s'exprime ainsi:}

À l'heure où les agents de l'éducation s'interrogent, parfois avec inquiétude, sur la portée, de caractère personnel et social, de l'enseignement de la philosophie dans les CEGEP québécois, cette publication devrait rejoindre les principaux intéressés. L'application de cette méthode ( . . . ) favorise plutôt le développement de la pensée personnelle: ce qui semble précieux, dans le contexte social actuel.

5. André Dagenais, Le Devoir, 4 nov. 1978. 


\section{DEUXIÈME PARTIE}

Critique de la méthode de recherche utilisée dans l'Expérience philosophique

Essentiellement, la critique de Lacoste porte sur l'hypothèse de départ qui instaurerait une polarisation entre la culture savante et la culture commune au sein de la méthode didactique, aboutissant à un dilemme.

\section{1 - Lacoste commence par affirmer:}

La faiblesse principale de l'Expérience philosophique, tient à sa tendance à définir le vécu de l'étudiant et son expérience comme un manque de culture savante.

\section{REMARQUES}

Nous ne croyons pas que l'on puisse décrire en termes de «tendance» l'élaboration d'un modèle théorique. Il est difficile de concilier ce terme avec l'objectivité scientifique et surtout la pratique expérimentale. En réalité, les choses se passent bien autrement.

Nous ne pouvons savoir, au début de nos recherches, comment se présente le comportement des étudiants relativement à la réalisation d'une tâche philosophique. Nous devons poser comme hypothèse qu'il est possible de connaître et d'identifier ce comportement relativement au comportement philosophique. Mais seuls les faits pédagogiques peuvent nous faire découvrir le comportement réel des étudiants, lors d'études préalables. Ces dernières constituent la phase la plus difficile et la plus longue de la recherche.

Car il ne s'agit pas de se baser sur des impressions, mais sur des faits observés et recueillis méthodiquement selon les exigences scientifiques. Il ne faut pas craindre de nous remettre en question, c'est-à-dire, notre méthode, notre conception de l'étudiant et notre conception de la philosophie.

Il s'agit de savoir ce qu'a fait l'étudiant en rapport avec la tâche et l'objectif pédagogiques. Nous ne savons pas, au départ, quelles sont les caractéristiques de ses réactions mentales, 
comment on pourrait les catégoriser. Si l'on parvient à catégoriser ces caractéristiques communes, on doit ensuite émettre une hypothèse expliquant ce phénomène. C'est ainsi que l'on peut obtenir un premier modèle théorique du fonctionnement mental de l'étudiant. Ce modèle théorique est donc une hypothèse.

Tenant compte de ce modèle, il s'agit d'émettre une nouvelle hypothèse sur une stratégie d'action en vue d'obtenir le comportement mental voulu. Ces stratégies d'action constituent à proprement parler un modèle didactique. On voit que ce modèle ne peut être gratuit, arbitraire: il est issu d'une symbiose entre le modèle théorique du fonctionnement mental de l'étudiant (qui se précise ainsi de plus en plus) et le modèle du comportement retenu comme objectif à atteindre.

Il s'agira de procéder ensuite à l'expérimentation en classe, dans les conditions requises. Si l'expérimentation révèle des résultats significatifs, non seulement les modèles seront valables et utiles, mais la théorie explicative elle-même prendra de la valeur.

On pourra, à partir de cela, produire des tests qu'il sera possible de vérifier de nouveau dans de nouvelles expérimentations. Lorsque la théorie est vérifiée, elle devient naturellement un acquis pour la didactique théorique. C'est là la pratique même de la didactique expérimentale, du moins en ce qui concerne l'enseignement de la philosophie. On voit bien qu'il ne saurait s'agir, dans cette optique, d'une "tendance», mais d'une exigence d'objectivité.

Nous avons consacré tout un chapitre (le premier de la première partie) à exposer ce que révèlent les faits pédagogiques relativement à l'expérience vécue. Le modèle obtenu au terme de cette recherche est didactiquement indiscutable. On n'a qu'à refaire en classe, auprès d'étudiants débutant en philosophie, la même expérience pédagogique et l'on arrivera aux mêmes résultats. Ce qui ne signifie pas que ce modèle de comportement de l'étudiant soit valable pour tous les domaines de la culture. Il ne vaut qu'en fonction de l'apprentissage philosophique. Quant à savoir le degré de culture savante de ces mêmes étudiants, il faudrait effectuer une recherche semblable en fonction des sciences ou d'une science en particulier. Seule, l'étude des faits pédagogiques pourrait trancher cette question d'un point de vue didacti- 
que, et non dans une perspective philosophique. Il faut donc conclure que cette affirmation de Lacoste, citée plus haut, se situe en dehors d'une perspective didactique.

2 - De plus, la conception que se fait Lacoste d'un modèle didactique semble étrangère à la pratique de la recherche expérimentale. C'est, du moins, ce qui se dégage de son argumentation:

Dans l'ensemble, écrit-elle, la prétention à définir des critères psychopédagogiques à partir des besoins réels des étudiants et sans définition préalable d'une méthode philosophique n'est que partiellement réussie. En réalité, c'est le modèle d'une culture savante qui préside à l'élaboration du modèle didactique, dans la mesure où l'expérience vécue apparaît comme défaut de précision, de systématicité, de profondeur, etc.

\section{REMARQUES}

Une méthode philosophique particulière implique un agencement des opérations mentales. Nous soutenons que l'hypothèse ne doit porter que sur les opérations et que ce sont les faits pédagogiques qui nous amèneront à définir le meilleur agencement relativement à des débutants en philosophie, en nous livrant les conditions réelles, concrètes de ces opérations (voir $\mathrm{p}$. 34). Ainsi, par exemple, nous croyions, au début de nos recherches, que la description d'une expérience vécue entrainaît presque automatiquement une analyse conceptuelle. Or les faits pédagogiques, c'est-à-dire les réactions mentales de nos étudiants, nous ont montré que tel n'était pas le cas. Il nous fallut émettre une autre hypothèse sur l'agencement des opérations.

Ce qu'il faut saisir ici, c'est que cette prétention à définir des critères psychopédagogiques à partir des besoins réels des étudiants sans définition préalable d'une méthode philosophique, n'exclut pas la nécessité d'une conception, préalable de la philosophie, comme nous le soulignons à la page 96 de l'Expérience philosophique:

Notre hypothèse de départ consistait à considérer la philosophie comme un retour réflexif sur l'expérience vécue.

Puis, nous ajoutions:

Les réactions mentales des étudiants nous ont peu à peu conduit à préciser de plus en plus ce procédé simple qui doit permettre 
d'atteindre le niveau de pensée que nous croyons être celui de la philosophie.

S'il s'agit ici, au départ, d'un modèle de culture savante, comme le prétend Lacoste, ce n'est sûrement pas un modèle de culture savante achevé. C'est plutôt un modèle qui se construit peu à peu, au cours de la recherche, comme nous l'expliquons:

L'hypothèse sert de point de départ pour obtenir des faits pédagogiques. Ceux que nous retenons aux fins de la science impliquent déjà un doute, une dérogation à la vision première contenue dans l'hypothèse, ou viennent confirmer celle-ci. Ce qui signifie que, souvent, un fait scientifique remet en cause une première hypothèse, incite à en proposer d'autres, de sorte qu'il devienne compréhensible, explicable. Et c'est ainsi que les hypothèses du début, devenues désuètes et inutiles, cèdent la place à d'autres qui expliquent mieux et plus adéquatement ( . . .) Au terme de ces recherches, nous pouvons nous faire une idée beaucoup plus exacte, quoique toujours passagère, jamais définitive, du mécanisme mental des étudiants, de même que du procédé philosophique (p. 96).

On n'a qu'à comparer le tableau de la page 107, schématisant les différents procédés, pour se rendre compte du chemin parcouru, relativement à l'hypothèse du début sur la philosophie. Il devient dès lors exagéré d'affirmer que c'est le modèle savant qui préside à l'analyse et à l'élaboration du modèle didactique. Que cette prétention, à laquelle Lacoste fait allusion, ne soit que partiellement réussie, seuls les faits pourraient le démontrer. Or je ne crois pas que l'argumentation suivante, que propose Lacoste, soit susceptible de le faire:

En outre, poursuit Lacoste, parce que le modèle didactique définit le processus de réflexion philosophique à partir d'une telle polarisation, on aboutit au dilemme, car la règle didactique accorde une priorité à l'étudiant comme point de départ à l'expérience philosophique tout en ayant tendance à l'identifier au phénomène de conscience naïve. C'est pourquoi, semble-t-il, les résultats auxquels les étudiants arrivent après avoir été l'objet de la démarche préconisée par Collin, laissent le lecteur perplexe. Dans l'ensemble, la démarche des étudiants semble aboutir à des travaux caractérisés par une plus grande généralité. Quant aux questions ultimes, elles ne transparaissent guère que par le biais d'une attitude moralisatrice, fortement personnalisée, mais plutôt courte.

Non seulement les bases, les prémisses de cette argumentation sont discutables, comme je crois l'avoir démontré plus haut, 
mais la preuve de fait proposée n'est pas valable. En effet, les travaux auxquels réfere Lacoste ne concernent que la première phase de la méthode didactique, que nous avons toujours qualifiée de préphilosophique.

Ce qu'il faut comprendre, c'est qu'un fait didactique doit être considéré dans sa double relation avec l'objectif poursuivi et la méthode utilisée. Or les faits mis en preuve sont en relation avec l'objectif pédagogique de «départiculariser» l'expérience (p. 78), et non de réaliser une expérience achevée. C'est pourquoi ils tendent tous à une plus grande généralité et se terminent souvent par un énoncé de principe.

Ces travaux démontrent que cette première phase doit être suivie d'autres phases destinées à permettre à l'étudiant d'atteindre un niveau de réflexion philosophique qui n'apparait, d'ailleurs, qu'à la troisième phase. (Voir les chapitres 2 et 3 de la première partie de l'Expérience philosopbique, où sont élaborées ces deux phases de la méthode didactique.)

Si Lacoste donne ainsi aux travaux en question une portée qu'ils ne sauraient avoir, c'est qu'elle a tendance à se situer dans une perspective philosophique plutôt que didactique. Ce qui nous permet de mieux comprendre plusieurs de ses affirmations. Bien qu'il soit légitime d'aborder un ouvrage de didactique expérimentale d'un point de vue philosophique, cela comporte un certain danger: on risque de projeter un éclairage qui fausse la véritable perspective de la didactique expérimentale, en soulevant des problèmes qui n'en sont pas vraiment.

3 - Lacoste termine ainsi sa critique:

On ne sait trop dans quelle mesure la méthode préconisée permettrait, en définitive, à l'étudiant d'accéder au pôle (valorisé) de la réflexion élaborée, systématique, achevée. On en vient à se demander si (...) le modèle didactique proposé par Collin n'aurait pas interdit, en cours de route, et didactiquement, de trop s'inquiéter de ce genre de question.

REMARQUES

Il est bien évident que seule une évaluation réalisée dans des conditions scientifiques requises serait en mesure de fournir une réponse à cette question fort légitime. Mais il ne faut pas trop anticiper. Beaucoup de travaux préalables devront être réalisés, 
concernant plus particulièrement le problème de la problématisation et aussi celui de la vérification philosophiques, avant de songer à entreprendre l'évaluation globale de la méthode didactique. Cela ne dépend pas du modèle didactique préconisé, mais de la méthode de recherche qui nécessite un nombre considérable de travaux préalables. Nous possédons suffisamment de données pour affirmer que l'évaluation des deux premières phases est techniquement possible actuellement, pourvu qu'il soit permis d'y procéder dans des conditions appropriées. Nous n'insistons pas sur ce point, tant que la recherche ne sera pas plus complète, concernant la dernière phase de la méthode didactique.

En guise de conclusion, nous voulons souligner jusqu'à quel point nous croyons que des échanges sereins et lucides concernant les nombreux problèmes que nous rencontrons dans l'enseignement de la philosophie peuvent contribuer à l'amélioration de notre enseignement. C'est dans cet esprit et animé d'un tel espoir que nous avons entrepris, il y a une dizaine d'années, avec la collaboration de Zdenko Osana, la tâche de proposer une méthode de recherche susceptible de favoriser l'élaboration d'une didactique objective de la philosophie.

Cette méthode de recherche, inspirée des travaux de Raymond Buyse ${ }^{6}$, en particulier, a fait l'objet de notre premier ouvrage, l'Enseignement de la philosophie. Il s'agissait, à nos yeux, de situer le problème didactrice dans l'ensemble de la pédagogie, d'exposer la méthode susceptible de l'étudier scientifiquement et de présenter les résultats d'une première expérimentation effectuée dans nos classes. Après six ans, nous constatons que les principales notions contenues dans ce premier ouvrage sont encore d'actualité: celle de fait pédagogique ${ }^{7}$, d'objectif pédagogique $^{8}$, de comportement mental ${ }^{9}$, d'autonomie relative de la didactique ${ }^{10}$, de transformation ${ }^{11}$, etc.; que la place que nous

6. Raymond Buyse, l'Expérimentation en pédagogie, Bruxelles, Maurice Lamertin, 1935.

7. Id., ibid.. p. 134 et suivantes; voir aussi Nathaniel Lees Gage, Handbook of Research on Teaching. Chicago, McNally, 1965, p. 48-51

8. Benjamin Samuel Bloom, éditeur, Taxanomie des objectifs pédagogiques, Montréal, Éducation Nouvelle, 1969 , p. 14-15.

9. E. Stones, An Introduction to Educational Psychology, London, Methuen, 1966, p. 52

10. Maurice Debesse et Gaston Mialaret, Traité des sciences pédagogiques, Paris, P.U.F., 1969 , tome 1, p. 44 et suivantes.

11. Jerome Seymour Bruner, The Process of Education. Vintage Books, New York, 1965, p $48-49$. 
assignions à la didactique au sein de la pédagogie, en accord avec les auteurs indiqués plus haut, est encore une donnée de base pour toutes les recherches en vue de l'élaboration d'une didactique objective. Mais ce qui me semble plus important, au-delà des notions qui sous-tendent cette méthode de recherche, c'est la possibilité qu'elle offre d'élaborer des modèles qui, eux, nous éclairent sur les conditions psychopédagogiques de notre enseignement.

C'est plus précisément ce que nous avons tenté de faire dans l'Expérience philosophique. Pour le lecteur averti, il est clair que la deuxième partie de cet ouvrage, portant sur les études concernant l'expérience vécue des étudiants, fonde l'élaboration du modèle de fonctionnement mental de l'étudiant, que nous exposons dans la première partie (théorique) ${ }^{12}$. Tout simplement, parce que ce sont les faits pédagogiques qui révèlent les conditons réelles de l'enseignement. C'est la raison pour laquelle ce modèle peut être utile à toute méthode pédagogique, quelle que soit la philosophie particulière mise de l'avant comme méthode d'exposition ou comme grille d'interprétation.

Tenant compte de ce modèle du fonctionnement mental de l'étudiant, l'étude des faits pédagogiques nous conduit à l'élaboration d'un modèle didactique constitué d'une triple mise en ordre de la réflexion, de telle sorte que nous avons l'impression d'avoir cerné l'activité «philosophante» de l'étudiant qui apprend à philosopher. L'objectif de l'enseignement de la philosophie devient alors le développement de cette activité philosophante; en d'autres termes, nous leur fournissons des moyens éprouvés d'élaborer leur propre philosophie.

Par la force des choses, l'utilisation de la méthode d'investigation scientifique préconisée dans le premier ouvrage, permet de mettre l'accent sur la "construction" plus que sur le "déjàconstruit». C'est pourquoi le second ouvrage se présente comme une étude de la "philosophisation", c'est-à-dire, des conditions psychopédagogiques de la réalisation de l'expérience philosophique.

Département de philosophie Collège du Vieux-Montréal

12. L'Expérience philosophique, p. 28 et suivantes. 